This item was submitted to Loughborough's Research Repository by the author.

Items in Figshare are protected by copyright, with all rights reserved, unless otherwise indicated.

\title{
Impact dynamics of rough and surface protected MEMS gears
}

PLEASE CITE THE PUBLISHED VERSION

http://dx.doi.org/10.1016/j.triboint.2008.05.019

PUBLISHER

(c) Elsevier

VERSION

AM (Accepted Manuscript)

LICENCE

CC BY-NC-ND 4.0

REPOSITORY RECORD

Teodorescu, M., Stephanos Theodossiades, and Homer Rahnejat. 2019. "Impact Dynamics of Rough and Surface Protected MEMS Gears”. figshare. https://hdl.handle.net/2134/13348. 
This item was submitted to Loughborough's Institutional Repository (https://dspace.lboro.ac.uk/) by the author and is made available under the following Creative Commons Licence conditions.

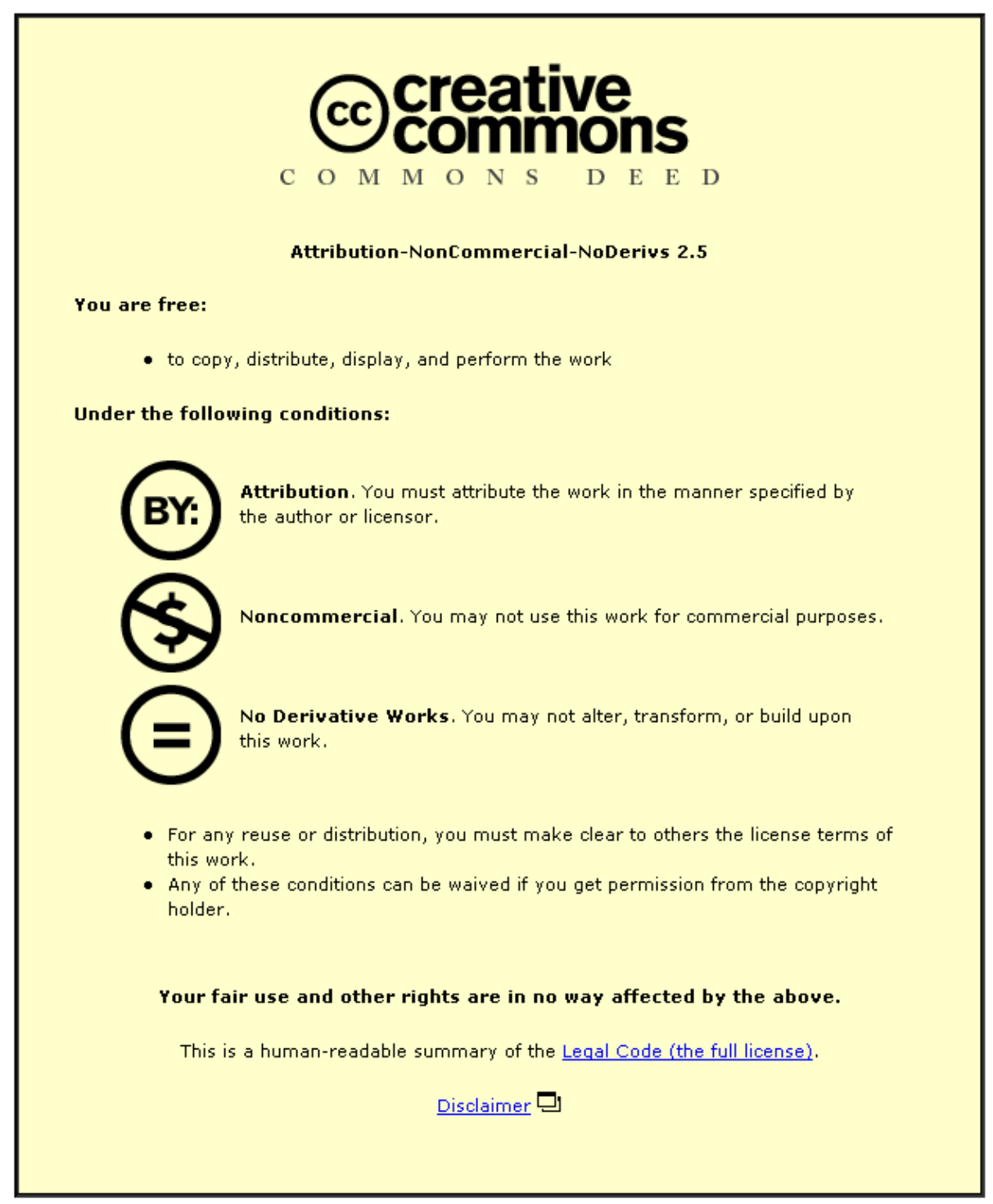

For the full text of this licence, please go to: http://creativecommons.org/licenses/by-nc-nd/2.5/ 


\title{
Impact dynamics of rough and surface protected MEMS gears
}

\author{
M. Teodorescu*, S. Theodossiades ${ }^{* *}$, and H. Rahnejat ${ }^{* *}$ \\ * School of Engineering, Cranfield University, UK \\ ${ }^{* *}$ Wolfson School of Mechanical and Manufacturing Engineering, Loughborough University, Loughborough, UK
}

\begin{abstract}
The paper provides an analysis of dynamics of micro-gear pairs, typically used in an assortment of microelectromechanical system (MEMS) devices. It includes a mathematical hierarchical model of the impact dynamics of meshing gear teeth. It comprises the nanoscopic effect of asperity tips' adhesion for relatively rough surfaces on a microscopic level (overall contact domain). The analysis is extended to the depletion of long chain molecule Self-assembled molecules (SAM) in impact behaviour of meshing gear-teeth pairs. The analyses show that for the usual high operating speeds of MEMS gears, due to high impact velocities, the role of asperity tips' adhesion is quite insignificant. However, the same is not true for lower impact velocities, which would occur under start-up, run-up to normal operating speeds or during deccelerative motions. The paper proposes a novel spectral-based approach to predict the degradation of the protective SAM layer between meshing teeth, while the mechanism is in continual relative motion.
\end{abstract}

Keywords: Impact dynamics, Adhesion, MEMS, SAM, nanoscopic contact mechanics

\section{Nomenclature}

a Contact area of an asperity

$b \quad$ Backlash half-width

$D_{i, j}^{k, l} \quad$ Influence coefficient matrix

$d \quad$ local deformation of the gear tooth

$e\left(\varphi_{1}\right)$ Static transmission error

$E_{1,2} \quad$ Young's modulus of elasticity

$E^{\prime} \quad$ Reduced elastic modulus of contacting solids

$h \quad$ Elastic gap shape

$h_{\min } \quad$ Minimum elastic separation

$h_{\text {ref }} \quad$ Minimum rigid gap

$I_{1,2} \quad$ Mass moments of inertia of gear pair

$M_{1} \quad$ Excitation moment on the pinion

$M_{2} \quad$ Resistance moment developed at the driven gear

$M_{1 h} \quad$ Harmonic component of the excitation moment

$n \quad$ Number of asperities

$N \quad$ Number of asperities in unit area

$P \quad$ Applied load on an asperity

$\bar{P} \quad$ non-dimensional applied load on an asperity

$P_{0}^{\beta} \quad$ Hertzian contact force per asperity

$P_{a}^{\beta} \quad$ Force of adhesion of an asperity

$p_{a s p(k, l)}$ Pressure at any location $\mathrm{k}, \mathrm{l}$ in the computational grid
$P_{c}^{\beta} \quad$ Pull-off force

$R_{1,2} \quad$ Base circle radii of gear pair

$r_{1,2} \quad$ Local principal radii of curvature of the meshing teeth

$r \quad$ Equivalent radius of curvature

$t$ Time

$T_{g} \quad$ Torque variation on gear due to contact force

$u \quad$ Dynamic transmission error

$v \quad$ Impact velocity

$W \quad$ Contact load

$x \quad$ Transverse contact direction

$z \quad$ Mean asperity height

$\beta \quad$ Asperity tip radius

$\delta \quad$ Local deformation of an asperity

$\bar{\delta} \quad$ non-dimensional local deformation

$\delta_{c}^{\beta} \quad$ Maximum extent of asperity extension (stretching)

$\gamma \quad$ Free surface energy

$\left[\begin{array}{l}\varphi_{1} \\ \varphi_{2}\end{array}\right]$ Rotational coordinates of the gear pair

$\sigma \quad$ RMS surface roughness

$v_{1,2} \quad$ Poisson's ratio of contacting solids

$\mu \quad$ Tabor parameter

$\varepsilon \quad$ Range of surface forces

$\Omega \quad$ Excitation frequency 


\section{Introduction}

In recent years, the microelectronics industry has experienced an unprecedented growth. The initial trend has been based on a growing demand for faster and smaller devices. This demand is also currently driven by the need for embedding ubiquitous computing power in every aspect of modern life. Silicon has become the foundation block for microelectronics, thus the justifiably used phrase: "the age of silicon". The increased demand for silicon components has triggered significant improvements in methods of fabrication. New techniques have allowed deposition of thin successive layers of silicon, as well as the cutting techniques with nano-scale precision. These have enabled manufacture of complete micro-scale interconnected structures, using techniques that resemble carving and printing rather than the traditional manufacturing methods. One beneficiary has been the emerging micro-electro-mechanical systems (MEMS) [1-3].

Most MEMS contain a significant number of micro-size components. Many of these components act as load bearing and torque transmitting members, usually at very high speeds. However, the minute size of these elements means that the inertial forces are also quite small, allowing them to withstand relative velocities and accelerations which are prohibitive for their macro-scale counterparts. Although the methodology for manufacturing these mechanisms has been relatively successful, the early design theories were based on principles which may be regarded as those applied at macrocosm, in relative terms. However, closer analyses suggest that these theories do not immediately extend to the case of micro-scale mechanisms, particularly with respect to contact/impact dynamics in gaps of molecular dimensions (nanoscopic interactions). Consequently, from the outset it has become clear that the fabrication of such components would rely, at least in parts, on an implicit acceptance of some empirical approach, which has clearly manifested itself in an inherent level of unreliability.

Macroscopic gears are cut using specialised techniques. However, after the initial cutting process, gear teeth are carefully polished and their contact surfaces treated for certain improved characteristics, usually related to tribological performance. In contrast, the comfort of post-cutting treatments is lost in the case of micro-size gears due to the complexity of the required processes and cost implications. These gears are cut out of fabricated media in-situ, therefore, their surface roughness and minimum separations (backlash) are directly proportional to the precision of the cutting technique used. In practice, this is in the nano-scale range. Thus, for the minute size of MEMS gears, contact between meshing teeth is actually relatively rough. This means that adhesion between individual asperities can play a significant role [4].

In nano-scale conjunctions molecular interactions cannot be ignored, nor surface energy effects, as well as the aforementioned adhesion. Furthermore, stiction by wetting action of any intervening fluidic media would also play an important role. In most cases, nano-scale conjunctions are prone to failure or mal-function due to structural degradation (wear and damage) of the load bearing and power transmitting conjunctions. This is usually due to adverse kinetic balance, principally caused by surface adhesion and ingression of moisture, giving rise to stiction [5]. Remedial solutions include measures against environmental humidity such as the introduction of selfassembled mono-layers (SAM), which adhere to the surfaces and are hydrophobic in nature. Additionally, such devices are maintained in sealed inert atmospheres, thus reducing the chance of ingressing condensation. These actions have proven to be relatively successful [6-9]. Thus, the current analysis is confined to the contact mechanics of asperity tips' interactions at nanoscopic level, when they are treated as hemispheres subject to the combined effect of Hertzian impact and adhesion. Therefore, the current analysis embodies the inherent assumption of very small strain elastic impacts.

This paper provides a numerical investigation of micro-dynamics of a pair of silicon gears (a pinion and a gear), incorporating detailed nano-scale impact dynamics of rough meshing gear teeth, protected with a self-assembled mono-layer of hydrophobic octadecyltrichlorosilane (OTS) long chain molecules. These develop reliable bonds with the silicon substrate and reduce the free surface energy. The mathematical model accounts for an assumed progressive degradation of the SAM layer (emulating gradual wear) and investigates its effect over the dynamics of the micro-gear assembly. The paper proposes a novel approach to ascertain the state of SAM layer during operating conditions. 


\section{The mechanical model}

Figure 1 shows an overview of the micro-gear system considered in this study. It consists of a pair of spur gears used in MEMS mechanisms. The purpose of this study is to highlight some of the particular characteristics of gear dynamics and contact mechanics of meshing gear teeth pairs. Therefore, a generic gear geometry is used.

MEMS gears are typically very small $(20-100 \mu \mathrm{m}$ outside diameter) and relatively slender; of the order of $2 \mu \mathrm{m}$ and quite rough (4-20 nm Ra) [9]. In macroscopic conventional gear pairs, the separation between the two meshing teeth ( $h_{r e f}$ in figure $1(\mathrm{~b})$ ) is orders of magnitude smaller than the tooth thickness. However, for MEMS, the manufacturing technique leads to separations that can be comparable to the tooth thickness itself (i.e. backlash of the order of $0.1 \mu \mathrm{m})$.

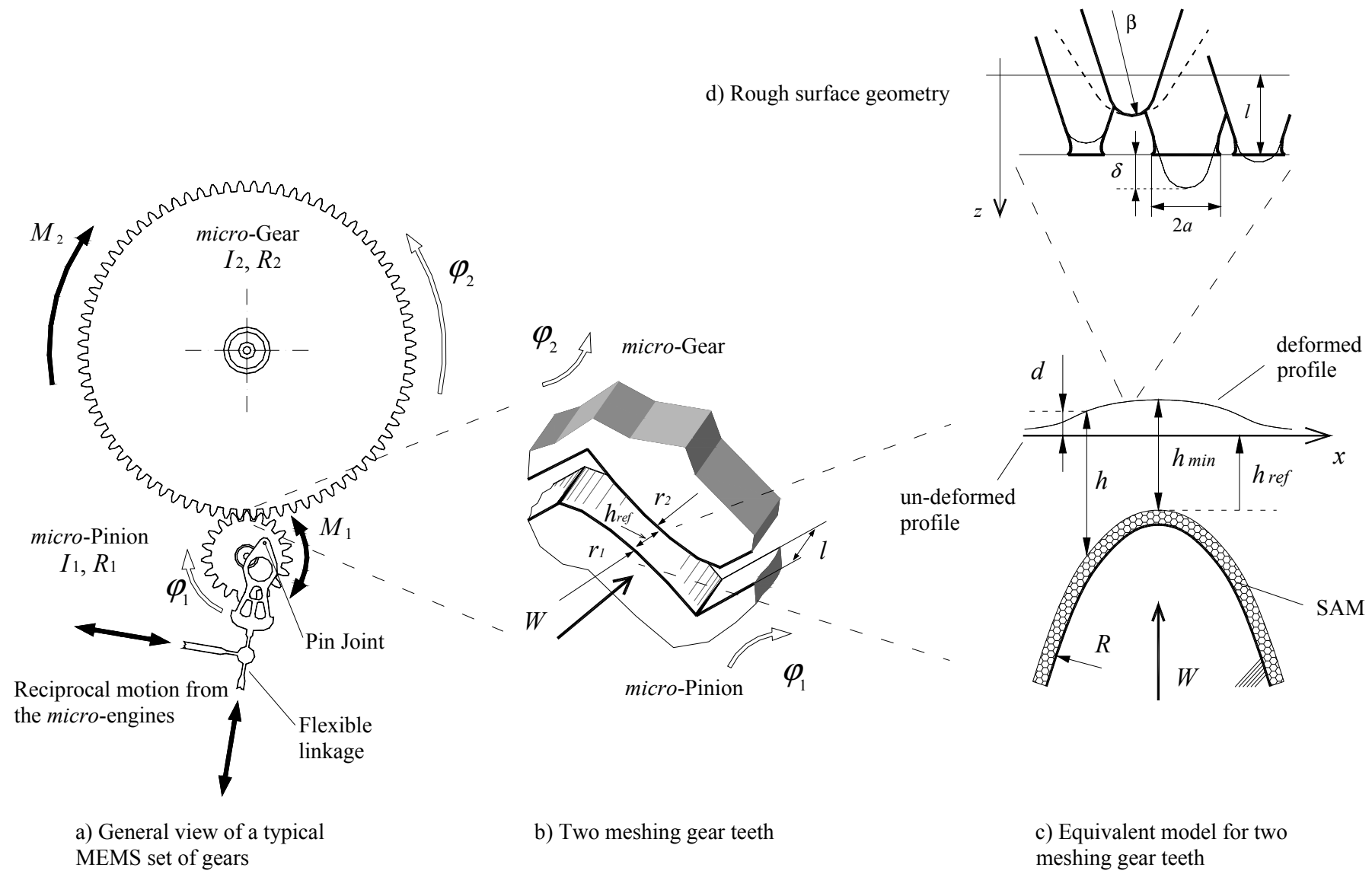

Figure 1: General overview of the MEMS gears and the modelling approach

\subsection{Micro-scale dynamics under impacting conditions}

The model comprises a pair of spur gears with mass moments of inertia $I_{n}$ and base radii $R_{n}$ (where $n=1,2$ represent the pinion and the gear respectively). Both gears are assumed to be supported by rigid shafts. The driving gear $(n=1)$ is subjected to a known torsional moment, $M_{1}$, whilst the driven gear $(n=2)$ develops a resistance moment, $M_{2}$.

The essential dynamics of the system can be described by a two-degrees of freedom torsional system, with coordinates $\left(\varphi_{1} \quad \varphi_{2}\right)^{T}[10,11]$.

The equations of motion for the two-degrees of freedom system can be expressed as: 


$$
\left\{\begin{array}{l}
I_{1} \ddot{\varphi}_{1}+R_{1} f_{g}\left(\varphi_{1}, u, \dot{u}\right)=M_{1}\left(t, \varphi_{1}, \dot{\varphi}_{1}\right) \\
I_{2} \ddot{\varphi}_{2}-R_{2} f_{g}\left(\varphi_{1}, u, \dot{u}\right)=-M_{2}\left(t, \varphi_{2}, \dot{\varphi}_{2}\right)
\end{array}\right.
$$

where $M_{1}\left(t, \varphi_{1}, \dot{\varphi}_{1}\right)$ represents the excitation moment on the pinion and $M_{2}\left(t, \varphi_{2}, \dot{\varphi}_{2}\right)$ the resisting moment developed at the driven gear.

The excitation torque applied to the pinion is generated by two reciprocal micro-engines mounted orthogonally to each other [1]. These engines are coupled to the same flexible linkage, which in turn is connected to the pinion through a pin-joint, mounted eccentrically with respect to the centre of the pinion. The pinion is spanned by conveniently operating the two micro-engines with a prescribed phase angle. Therefore, the moment applied on the pinion has an oscillatory behaviour about an equilibrium position. To capture this characteristics, in the current analysis the excitation moment is given a harmonic component, superimposed upon a constant steady state value.

$$
\begin{aligned}
& M_{1}\left(t, \varphi_{1}, \dot{\varphi}_{1}\right)=M_{10}+M_{1 h} \operatorname{Cos}(\Omega t) \\
& M_{2}\left(t, \varphi_{2}, \dot{\varphi}_{2}\right)=M_{20}+\alpha\left(\dot{\varphi}_{2}\right)^{2}
\end{aligned}
$$

When $\alpha \neq 0$, the numerical results are obtained by selecting an appropriate range of values for the driving moment $M_{10}$. For these cases, it turns out that under steady state conditions [12]:

$$
M_{10}=\frac{R_{2}}{R_{1}}\left[M_{20}+\alpha\left(\dot{\varphi}_{2}\right)^{2}\right]
$$

where $\dot{\varphi}_{2}$ is the average gear rotational speed.

This model can also take into account the static transmission error, $e\left(\varphi_{1}\right)$, representing geometrical errors of the gear teeth profiles and spacing. In addition, the dynamic transmission error defined below is determined as:

$u=R_{1} \varphi_{1}-R_{2} \varphi_{2}-e\left(\varphi_{1}\right)$

$u$ is known as the dynamic transmission error. However, for simplicity, in this analysis $e\left(\varphi_{1}\right)$ is considered to be negligible.

Taking into account the backlash, the damping mechanism and the restoring force component in the gear mesh may be expressed in a simple form as:

$$
f_{g}\left(\varphi_{1}, u, \dot{u}\right)=F_{g}\left(h_{r e f}, h, \dot{h}\right)
$$

where $2 b$ represents the total gear backlash.

The rigid separation $h_{r e f}$ in figure 1(c) can be expressed as:

$h_{\text {ref }}= \begin{cases}b-u, & R_{1} \varphi_{1}>R_{2} \varphi_{2} \\ b+u, & R_{1} \varphi_{1}<R_{2} \varphi_{2}\end{cases}$

\subsection{Contact mechanics of meshing teeth}

Referring back to figure 1, note that the contact of a pair of rough teeth is treated at two levels. At the microscopic level (figure $1(\mathrm{c})$ ), the overall contact domain is determined, in which clearly a number of asperities exists, whose 
interactions are determined by their nanoscopic tip contacts according to a computed gap shape between the approaching contiguous solids. In normal orthogonal contact of hemispherical asperity tips of radii $\beta$ (figure 1(d)), their combined deformation and adhesion should be considered under the assumed inert atmosphere. The asperities have molecularly smooth surfaces, even though the actual contact is quite rough. Thus, friction at the nanoscopic level may be neglected. The same, of course, is not true of the microscopic contact, where in the presence of relative surface velocities friction would exist. Friction is generated both in breaking adhesive junctions and through ploughing. These mechanisms of friction are very significant for micro-scale roughness, where an appropriate model is proposed by Greenwood and Tripp [13], where asperity deformation is based on the classical Hertzian theory. In nano-scale asperity interactions are governed by combined deformation and adhesion.

At the microscopic level, the impact of meshing gear teeth can be approximated by a roller of an equivalent radius to that of the local principal radii of curvature of the meshing teeth as: $1 / R=1 / r_{1}+1 / r_{2}$ against a semi-infinite elastic half-space of reduced elastic modulus: $E^{\prime}=2 /\left[\left(1-v_{1}^{2}\right) / E_{1}+\left(1-v_{2}^{2}\right) / E_{2}\right]$. Figure 1 (c) shows the equivalent configuration for the impacting conditions. $h_{r e f}$ is the nominal separation between the roller and the undeformed flat frictionless plane, $h_{\min }$ is the minimum elastic separation, $h$ is the local separation and $d$ is the local deflection [14].

This set of approximations represents the basis for the classical Hertzian impact problem. However, for rough contacts with nano-scale gaps asperity adhesion also plays a significant role. Therefore, the contact behaviour deviates from the Hertzian theory. If moisture would be present in the contact, a combination of inertial, adhesion, hydration and nano-meniscus forces can also contribute [5]. To include the contribution due to adhesion a modified version of the Hertzian approach is required. Currently there are two classical models which predict the deformation and adhesion of dry indenters on smooth substrates. One was proposed by Johnson et al. [15, 16] (JKR) and extended by Fuller and Tabor [17] for surfaces with normally distributed asperities. The other asperity contact model was proposed by Derjaguin et al. [18] (DMT). The JKR model is applicable to more compliant asperities with strong adhesion, whilst DMT is more suited to stiffer asperities with weak adhesion (with the intermediate range covered by the model proposed by Maguis [19]). To differentiate between these two classical models it is customary to compute the Tabor parameter $[20,21,22]$ as: $\mu=\left(\beta^{1 / 3} \Delta \gamma^{2 / 3} /\left[E^{\prime} / 2\right]^{2 / 3} \varepsilon\right)$, where $\varepsilon$ is the acting range for the surface forces [22] and $\Delta \gamma=\gamma_{1}+\gamma_{2}+\gamma_{12}$. As the Tabor parameter shows the significance of adhesion depends on free surface energy and asperity geometry. This is more significant in nanoscale roughness. Therefore, for the JKR approach to be used $\mu$ should be larger than 5 . Therefore, using Tabor's formulation, the surface roughness should be below $50 \mathrm{~nm}$ for unprotected silicon surfaces and $8 \mathrm{~nm}$ for the same surfaces protected by SAM. The nanoscopic contact of the hemispherical asperities is regarded as smooth, thus the use of JKR model is justified with Fuller and Tabor's approach. Hence:

$$
P^{\beta}(z)=P_{0}^{\beta}-P_{a}^{\beta}=\frac{4 E^{\prime} a^{3}}{3 \beta}-\sqrt{8 \pi a^{3} \Delta \gamma E^{\prime}}
$$

After a contact is established, the removal of the initial load leads to the diminution of the contact force. To separate an asperity with the undeformed radius $\beta$, a pull-off force of $P_{c}^{\beta}=1.5 \pi \beta \Delta \gamma$ is required and a maximum asperity extension (stretching) of $\delta_{c}^{\beta}=\left(9 \beta P_{c}^{\beta} / 4 E^{\prime}\right)^{2 / 3} / 3 \beta$ would be required. For the current contact conditions: $\gamma_{1}=\gamma_{2}$ and for relatively smooth surfaces it can be assumed that: $\gamma_{12}=0$. Adhesion of asperities manifests itself under any contact condition, but its effect can be observed mainly with very small scale asperities.

For ease of implementation in a computer code, it is customary to use equation (5) in a non-dimensional form:

$$
\bar{P}=2\left(\bar{a}^{3}-\sqrt{2 \bar{a}^{3}}\right) \quad \bar{\delta}=\sqrt[3]{3^{2}} \cdot \bar{a}^{2}\left(1-\frac{2 \sqrt{2}}{3} \bar{a}^{-\frac{3}{2}}\right)
$$


The above expounded approach predicts the contact condition between two approaching hemispherical asperities. For two rough surfaces in contact many asperity-pairs can be involved simultaneously. Therefore, the statistical distribution of these asperities should to be taken into account. Considering a normally distributed asperity tips, the probability that an asperity has a height between $z$ and $z+d z$ above the plane, defined by the mean asperity height, is usually given as:

$$
\phi(z)=\frac{1}{\sigma \sqrt{2 \pi}} e^{-z^{2} / 2 \sigma^{2}}
$$

Similarly, considering a normal distribution for asperity tip radius, the probability of an asperity having a radius between $\rho$ and $\rho+d \rho$ can be given as:

$\psi(\rho)=\frac{2}{\beta \sqrt{2 \pi}} e^{-z^{2} / 2 \beta^{2}}$

If the unit area is covered with $N$ asperities, the number of asperities with the height between any arbitrary limits $z_{1}$ and $z_{2}$ and curvature radius between $\rho_{1}$ and $\rho_{2}$ is:

$$
n_{z_{1} \div z_{2}}^{\rho_{1} \div \rho_{2}}=N \int_{z_{1}}^{z_{2}} \phi(z) d z \int_{\rho_{1}}^{\rho_{2}} \psi(\rho) d \rho
$$

The pressure due to the asperity interactions can thus be expressed as:

$p_{a s p}=N \frac{P_{c}^{\mathrm{B}}}{\sqrt{2 \pi}} \int_{-L}^{\infty} f n\left(\frac{\Delta}{\Delta_{c}}\right) \exp \left\{-\frac{1}{2}(h+\Delta)^{2}\right\} d \Delta$

where:

$$
P_{c}^{\mathrm{B}}=1.5 \pi \mathrm{B} \Delta \gamma ; \mathrm{B}=\sqrt{\frac{2}{\pi}} \int_{0}^{\infty} \exp \left(-\frac{\rho^{2}}{2 \beta^{2}}\right) d \rho ; \Delta=\frac{\delta}{\sigma} ; \quad \Delta_{c}=\frac{\delta_{c}^{\beta}}{\sigma} ; \quad h=\frac{l}{\sigma}
$$

The lower integration limit correlates with the history of contact loading-unloading cycle. This is fully described in [4]. If the action previously compressing an asperity is removed, then the asperity may stretch and apply a negative force (adhesion). Therefore, to account for the stretched asperities, the integration limit has to be adjusted at each computational time step.

Equation (12) predicts the total pressure between two rough plane surfaces separated by a known gap [17]. For a roller of radius $R$, the local gap at any position in the contact is given in a computational grid as:

$$
h=\frac{x^{2}}{2 R}+h_{r e f}+d
$$

where the local deformation is obtained as [14]:

$$
d_{i, j}=\frac{1}{\pi E^{\prime}} \sum \sum D_{i, j}^{k, l} p_{a s p(k . l)}
$$


where $D_{i, j}^{k, l}$ is the influence coefficient matrix, determining the local deflection at a point $(i, j)$ as a result of a pressure contribution at a point $(k, l)$ (see Hamrock [23]).

Although the set of equations (12-14) is relatively simple, it is not possible to obtain a direct solution, which would determine the deformed gap shape $h$, as well as the instantaneous impact force for a given rigid approach $h_{\text {ref }}$. This drawback can be overcome using an iterative method. When the gap is relatively large, the contact pressure is negligible. Therefore, there is no deflection (equation (12)), and the rigid gap is parabolic (equation (13), where $d=0$ ). For a diminishing gap some of the asperities interact and the resultant pressures (equation (12)) cause elastic deformation of asperity with small strains in accord with the classical Hertzian theory. In turn, the resultant deformed gap gives rise to an increased pressure distribution which should be recomputed. After several iterations, the correct equilibrium between the deformed gap shape and the corresponding pressure distribution is reached.

Finally, the net impact force is obtained as:

$W=\sum_{k} \sum_{l} p_{a s p(k . l)}$

\section{Results and Discussion}

Before proceeding to the case of repetitive impact of gear teeth pairs and micro-dynamics of the gears, it is necessary to study the impact of a pair of teeth with and without SAM. Figure 2(a) shows the impact characteristics of a silicon roller of radius $5 \mu \mathrm{m}$ and mass $0.6 \mu \mathrm{g}$ on a silicon substrate, as well as the same pair protected by SAM. For the current model it was considered that for two silicon asperities $\gamma_{1}=\gamma_{2}=1.5\left[\mathrm{~J} / \mathrm{m}^{2}\right] \underline{\text { and }}$ for two asperities protected by SAM $\gamma_{1}=\gamma_{2}=0.1\left[\mathrm{~J} / \mathrm{m}^{2}\right]$. The peak impact force occurs at the maximum deflection, followed by rebound. With the rough unprotected silicon surfaces a negative force is encountered. This indicates contribution due to adhesion of the surface asperities [1]. This force has to be overcome in order for separation to occur. With SAM protected surfaces very small adhesive contribution is noted regardless of the impacting velocity. When the same analysis is carried out at high impacting velocities (see figure 2(b)) the adhesive characteristics are no longer noted and insignificant compared to the applied inertial force. These findings explain observations that operation of MEMS devices at low speeds can often lead to failure [1], one reason may well be due to adhesion, with another being formation of micro-menisci if condensation activation time is exceeded when protective sealing against normal atmospheric conditions is breached.

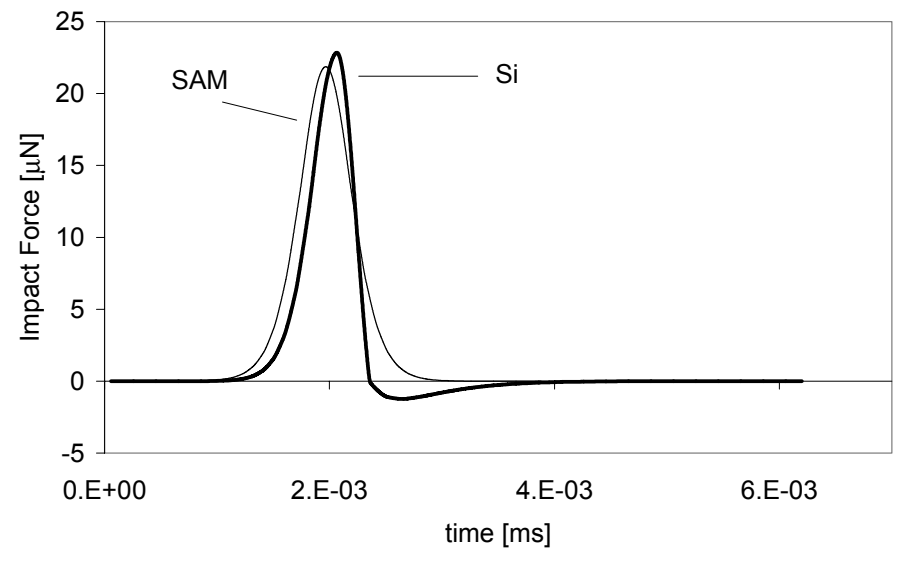

a) Low impact velocity $\left(v=10^{-2}[\mathrm{~m} / \mathrm{s}]\right)$

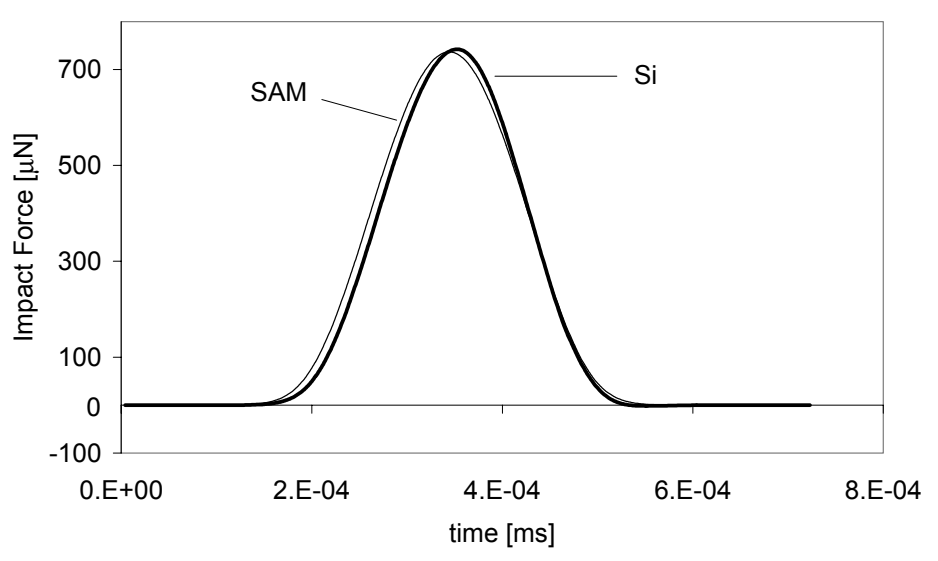

b) High impact velocity $\left(v=10^{-1}[\mathrm{~m} / \mathrm{s}]\right)$

Figure 2: Impact Force characteristics of a rough silicon roller with and without SAM 


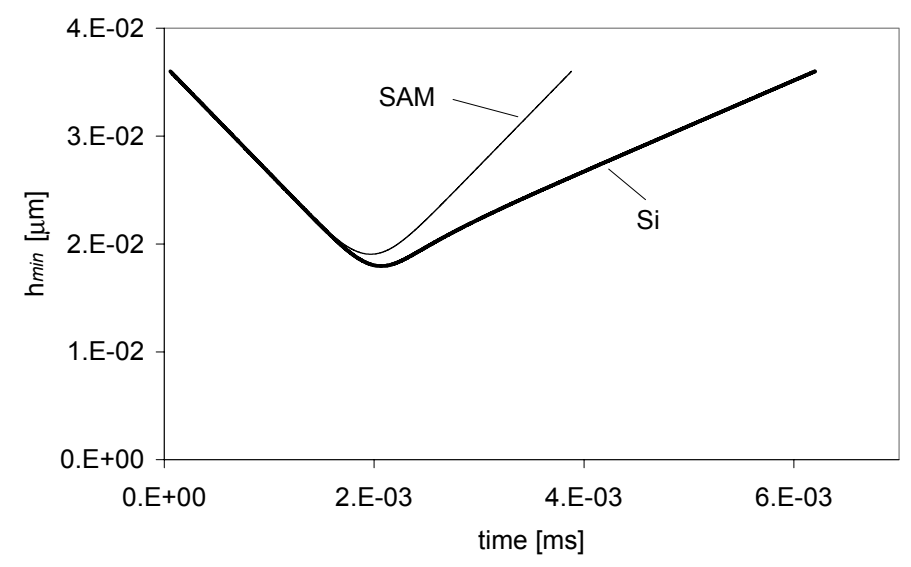

a) Low impact velocity $\left(v=10^{-2}[\mathrm{~m} / \mathrm{s}]\right)$

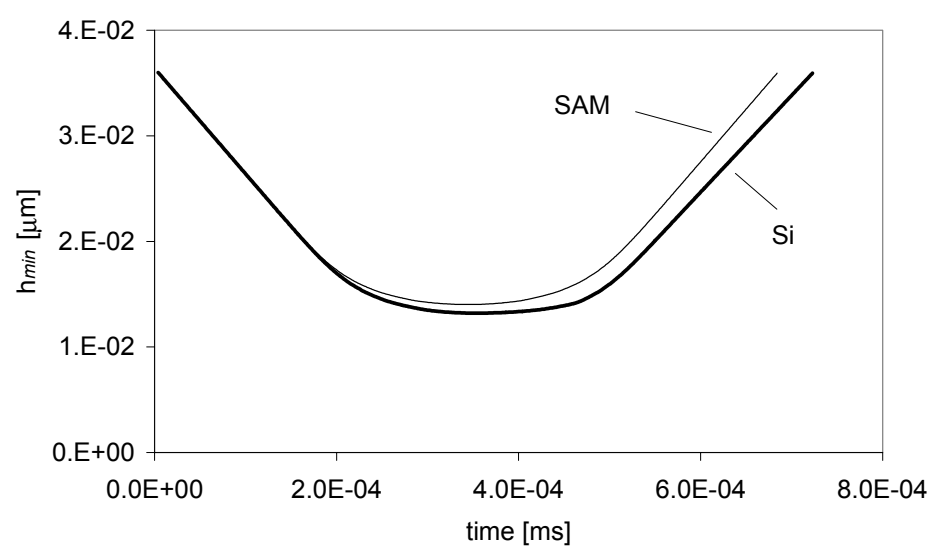

b) High impact velocity $\left(v=10^{-1}[\mathrm{~m} / \mathrm{s}]\right)$

Figure 3: Transient approach and rebound characteristics of a rough silicon roller with and without SAM

Figures 3(a) and (b) show the maximum contact deformation (the minimum elastic separations) during impact and rebound, corresponding to the impact force variations in figures 2(a) and (b) respectively. The surfaces separate at a slower rate in the presence of adhesion at lower impact and rebound velocities. It is, therefore, noteworthy that at even lower velocities, for example in a start-up process in micro-gear operations, circumstances may emerge that excessive adhesion would seriously jeopardise system operation. The adhesion effect is even noted at the usual operational speeds (see figure 3(b)), even though this is quite small.

Now returning to the micro-dynamics of the gear pair, incorporating the described contact model, one or two pairs of teeth are in simultaneous contact at any instant of time. The relevant geometrical, physical and operational data for the gearing pair are given in table 1.

\begin{tabular}{|c|c|c|c|c|c|c|c|c|c|}
\hline $\begin{array}{c}I_{1} \\
\left(\mathrm{Kgm}^{2}\right)\end{array}$ & $I_{2}\left(\mathrm{Kgm}^{2}\right)$ & $\begin{array}{c}R_{1} \\
(\mu \mathrm{m})\end{array}$ & $\begin{array}{c}R_{2} \\
(\mu \mathrm{m})\end{array}$ & $\begin{array}{c}h_{r e f} \\
(\mu \mathrm{m})\end{array}$ & $\begin{array}{c}M_{10} \\
(\mathrm{Nm})\end{array}$ & $\begin{array}{c}M_{20} \\
(\mathrm{Nm})\end{array}$ & $\begin{array}{c}M_{2 h} \\
(\mathrm{Nm})\end{array}$ & $\alpha$ & $\begin{array}{c}\Omega \\
(\mathrm{Hz})\end{array}$ \\
\hline $\begin{array}{c}1.5263 \mathrm{E}- \\
20\end{array}$ & $\begin{array}{c}6.1052 \mathrm{E}- \\
20\end{array}$ & 34.7 & 138.86 & 0.1 & $2.43 \mathrm{E}-11$ & $3 . \mathrm{E}-11$ & $0.3 \mathrm{E}-11$ & $\begin{array}{c}8 . \mathrm{E}- \\
18\end{array}$ & 10,000 \\
\hline
\end{tabular}

\begin{tabular}{|c|c|c|c|c|c|c|c|}
\hline $\begin{array}{c}E_{S i} \\
\left(\mathrm{~N} / \mathrm{m}^{2}\right)\end{array}$ & $\begin{array}{c}E_{S A M} \\
\left(\mathrm{~N} / \mathrm{m}^{2}\right) \\
{[7]}\end{array}$ & $v_{S i}$ & $v_{S A M}$ & $\begin{array}{c}N \\
{[23]}\end{array}$ & $\sigma(\mathrm{nm})$ & $\begin{array}{c}\text { Number } \\
\text { of teeth } \\
\text { (pinion) }- \\
{[1]}\end{array}$ & $\begin{array}{c}\text { Number } \\
\text { of teeth } \\
(\text { gear })- \\
{[1]}\end{array}$ \\
\hline $161 \mathrm{E} 9$ & $2 \mathrm{E} 9$ & 0.23 & 0.4 & $169.0 \mathrm{E} 14$ & 6 & 19 & 76 \\
\hline
\end{tabular}

Table 1: Physical data used in the model

Figure 4 shows the angular velocity of the gear under steady state conditions for two ideal cases. Firstly, the SAM is assumed to remain intact (100\% SAM) and secondly the layer is assumed to have completely perished (Si). In both cases the mechanism was excited by an identical torque. Although for the case of an intact SAM the mechanism operates at slightly higher speed, it is noticeable that once at high speed, the mechanism can operate in steady state conditions even in the theoretical case of fully unprotected gears. Its rather complex periodic nature is due to various spectral contributions, see later (figure 6). One cycle of such oscillations is shown in figure 5 . The instances of impact are shown by high frequency reversals in the figure. The longest periodic oscillation (at $10,012 \mathrm{~Hz}$ ) pertains to the carrier frequency due to imposed forcing. The other shorter periods correspond to the meshing frequency $(38,813 \mathrm{~Hz})$ and its harmonics due to the non-linear nature of the impact phenomenon. These are all shown in the power spectral density of figure 6. 


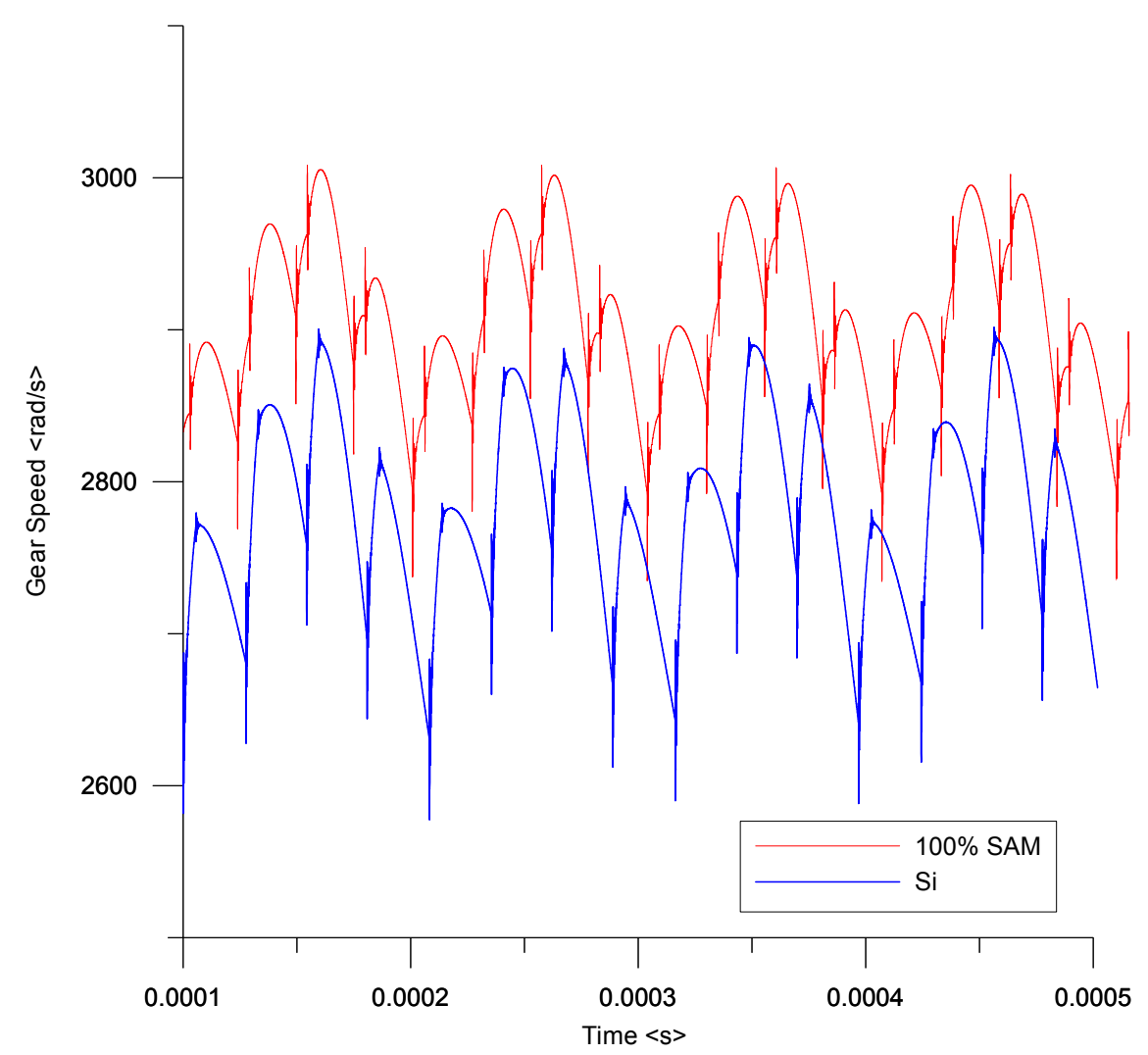

Figure 4: Angular velocity of the gear $\dot{\varphi}_{2}$ at high speed under steady state condition

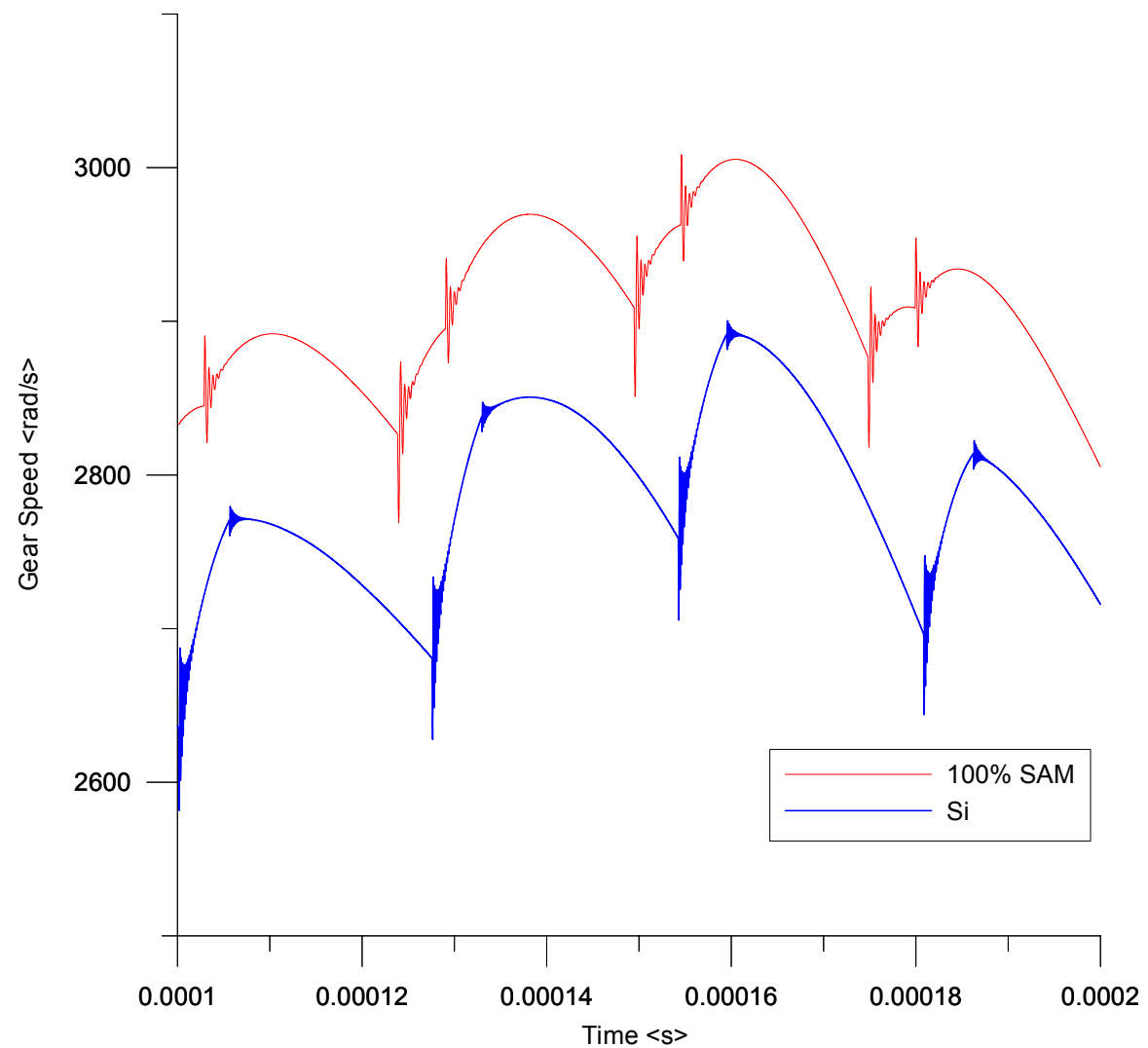

Figure 5: One cycle of the angular velocity of the gear $\dot{\varphi}_{2}$ at high speed under steady state condition 


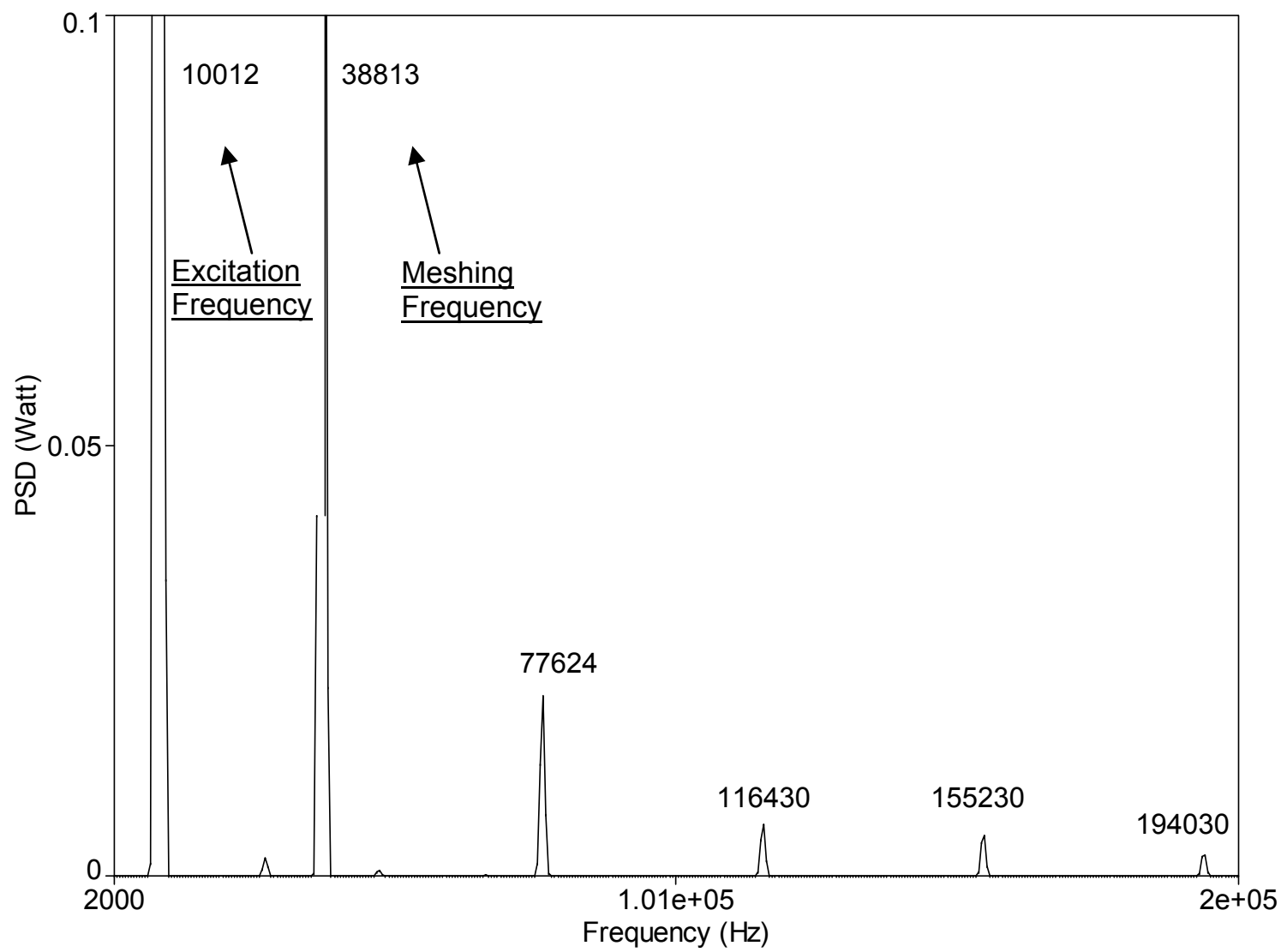

Figure 6: Power spectral density of the gear rotational velocity $\dot{\varphi}_{2}$

One of the accepted methods to investigate the reliability of SAM coatings, as well as MEMS mechanisms as a whole, is to alternate the excitation from high torque to low torque. This operates the mechanism successively at high speed (normal operating conditions) and at low speed (approximately 1Hz). Tanner et al. [1] noticed that in the initial stages the engine has a smooth operation at high speed as well as at low speed. However, following the this initial period the behaviour progressively deteriorated, when running at low speed, but remained smooth at high speed. It is understood that the main cause of this behaviour is the progressive wear of SAM. In the initial stages, SAM covers the contacting surfaces in an uninterrupted manner. This ensures low adhesion and smooth operation under all conditions. However, after the SAM has partly worn out or perished, the exposed sections of the contact are subject to higher adhesion. This affects the operation of gears at low speed as shown in figures 2 and 3, when the inertial dynamics has to overcome a higher adhesive force. In practice, if the SAM is damaged, a smaller proportion of the impacting surfaces are protected and the adhesive forces during the rebound increase.

To understand the implications of operating the set of gears at low speed, the model was run for a very low excitation torque. Figure 7 shows the results for different assumed percentages of SAM wear. It should be noted that at low speed the gears operate smoothly if the layer is continuous (100\% SAM), and quite erratically if only $50 \%$ of the layer remains intact. The current model assumes uniform wear of the SAM protective layer. Therefore, equal proportion was assumed in calculation of adhesion contributions for exposed (worn) and protected areas. However, if the layer is even further damaged, the mating pairs tend to stick. The high vibrations in the initial section of figure 7 are transient effects due to initial conditions in numerical simulation. Some conclusions can be made of the simulation results thus far, which agree with reported observations. One conclusion is that if adequate protection against atmospheric conditions can be assured, for gears running at high speed, the effect of adhesion would be rather insignificant and a pair of unprotected silicon surfaces would perform equally as well as those furnished with SAM. However, the same cannot be claimed at start-up or run-up to the normal operational speeds or during braking to lower speeds where reduced impact velocities may be encountered. For the cases studied here the most likely failure modes may be cyclic fatigue due to impact loads. Future analysis should consider cyclic fatigue at high operational speeds and adhesion contributions at lower speeds and higher impact durations. It should be noted that in the current analysis the localised wear and damage has not been the subject of investigation. 


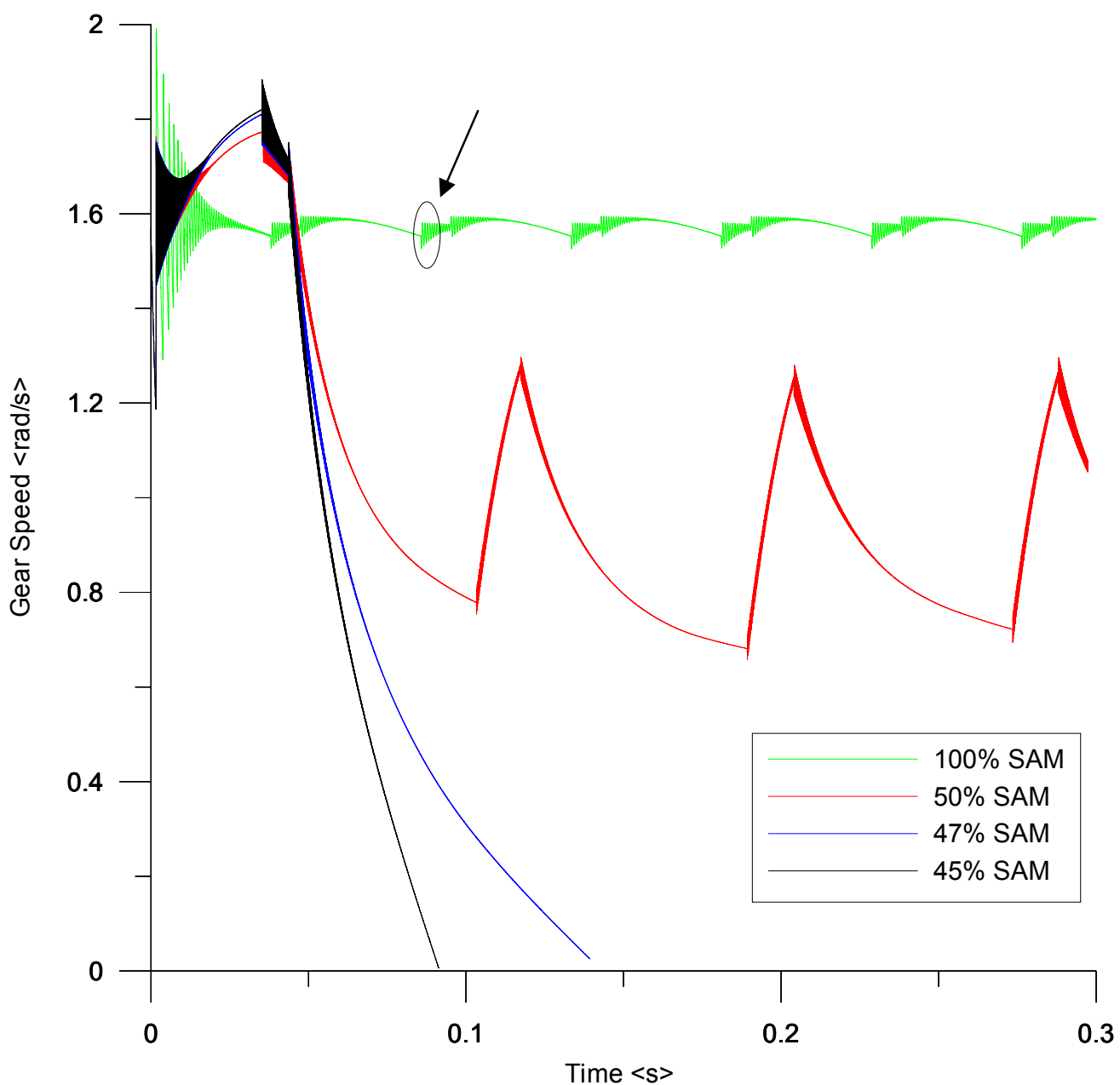

Figure 7: Angular velocity of the gear $\dot{\varphi}_{2}$ at low speed for different degrees of SAM wear

Figure 8 shows the torque variation on the gear introduced by the contact/impact force under the same conditions as those described for figure 7 . The sudden torque reversals shown are responsible for the corresponding sharp angular velocity variations in figure 5 . These torque reversals occur in pairs since two pairs of quickly successive impacts occur in cycles of meshing. The impact forces correspond to the typical high velocity impacts of figure 2(b). It should be noted that for the case of a pair of gears with an intact protective layer (100\%SAM in figure 8), the torque is positive regardless of the impacting conditions. However, if the layer is damaged and significant adhesion takes place between the contacting asperity pairs, the torque applied on the gears can be either positive or negative. When the SAM wears out, the adhesion component increases. At low impact velocities this component can overcome the rebound force and consequently the two surfaces tend to stick.

Referring back to figure 7 , the time history for the case of $100 \%$ SAM can be windowed around the sharp oscillations pertaining to impact and rebound response of the rough teeth-pairs. A Fourier analysis can be carried out for this windowed domain (the procedure referred to as short FFT), resulting in the spectrogram shown in figure 9. This indicates a dominant peak, although much smaller contributions also exist. This peak is the characteristic response of the contact mechanics behaviour, which would alter according to percentage presence of SAM and the material of the substrate, clearly silicon in this case. For the impacting surfaces of the same substrate material combination, geometry and surface topography the only variable would be the state of SAM coverage that affects this characteristic frequency for a given excitation input torque. Therefore, a series of spectrograms of simulation results, such as those shown in figure 7 would yield the variation of the characteristic impact frequency with SAM coverage of the contacting surfaces as shown in figure 10 (the characteristic response for the case of figure 9 is indicated as a point on the graph). Such a graph can have its useful purpose in monitoring the state of health of SAM covered surfaces as a preventative tool. The speed of gear can be monitored and a spectrogram obtained on line. The characteristic frequency, together with a graph such as that in figure 10, would indicate the state of SAM coverage. It should be noted that the graph of figure 10 is but one of a series of such lines that would vary according to the input torque and the type of SAM used. Clearly, the model would require further refinement to be of direct practical use, but in its current state represents a reasonable base for developments to follow. 


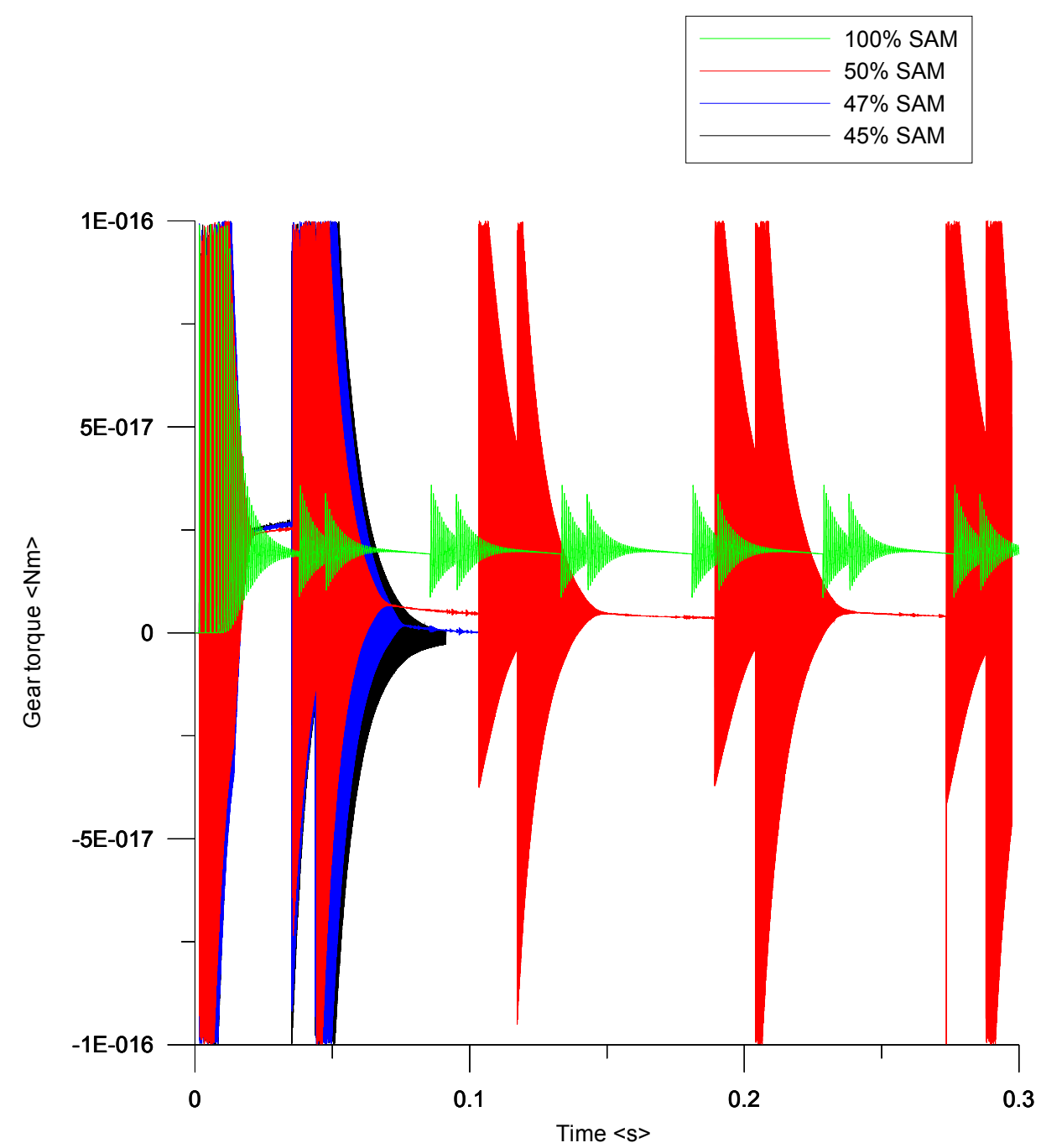

Figure 8: Torque variation on the gear introduced by the contact force

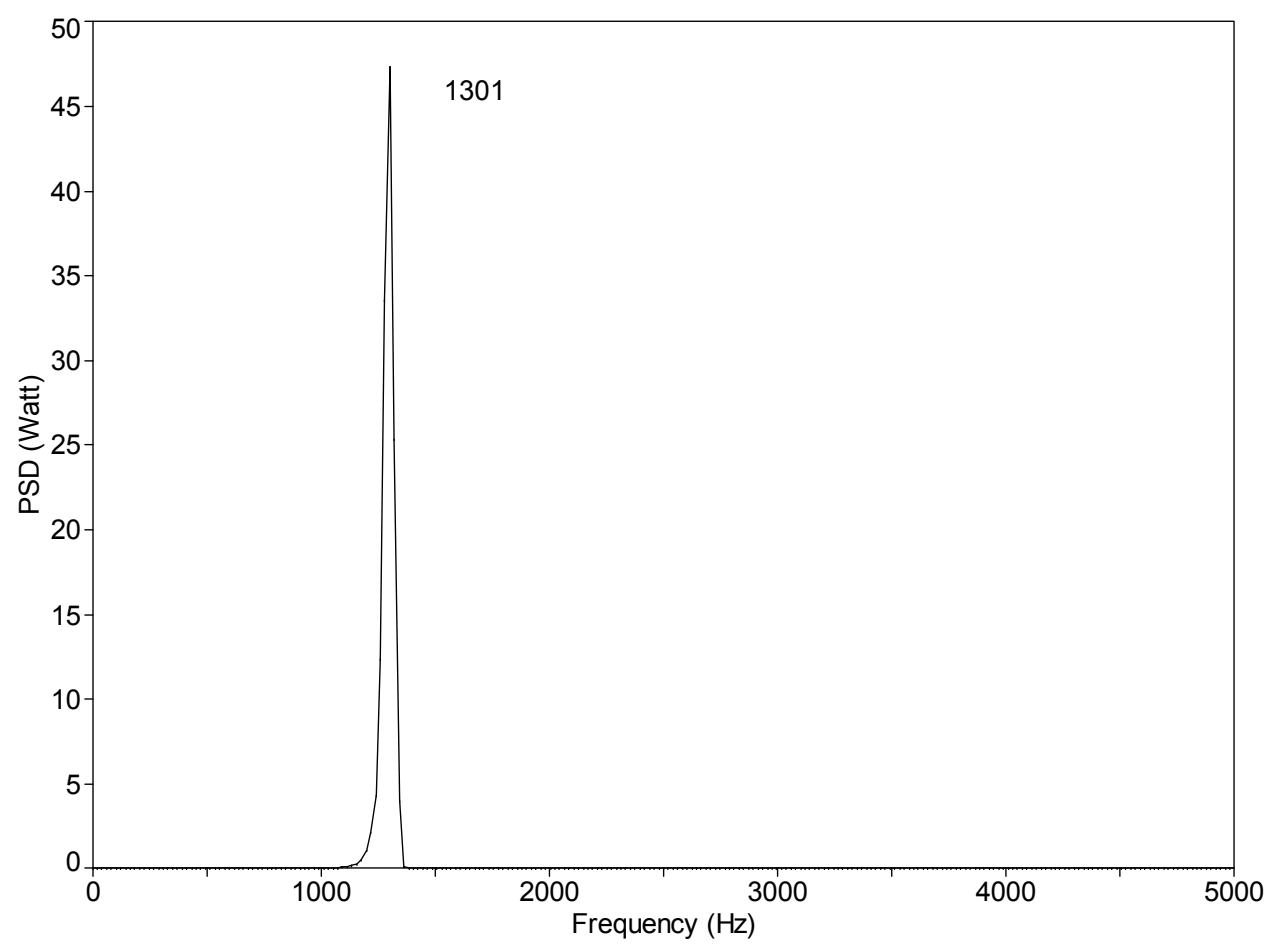

Figure 9: A spectrogram of power spectral density for impact event window of figure 7 


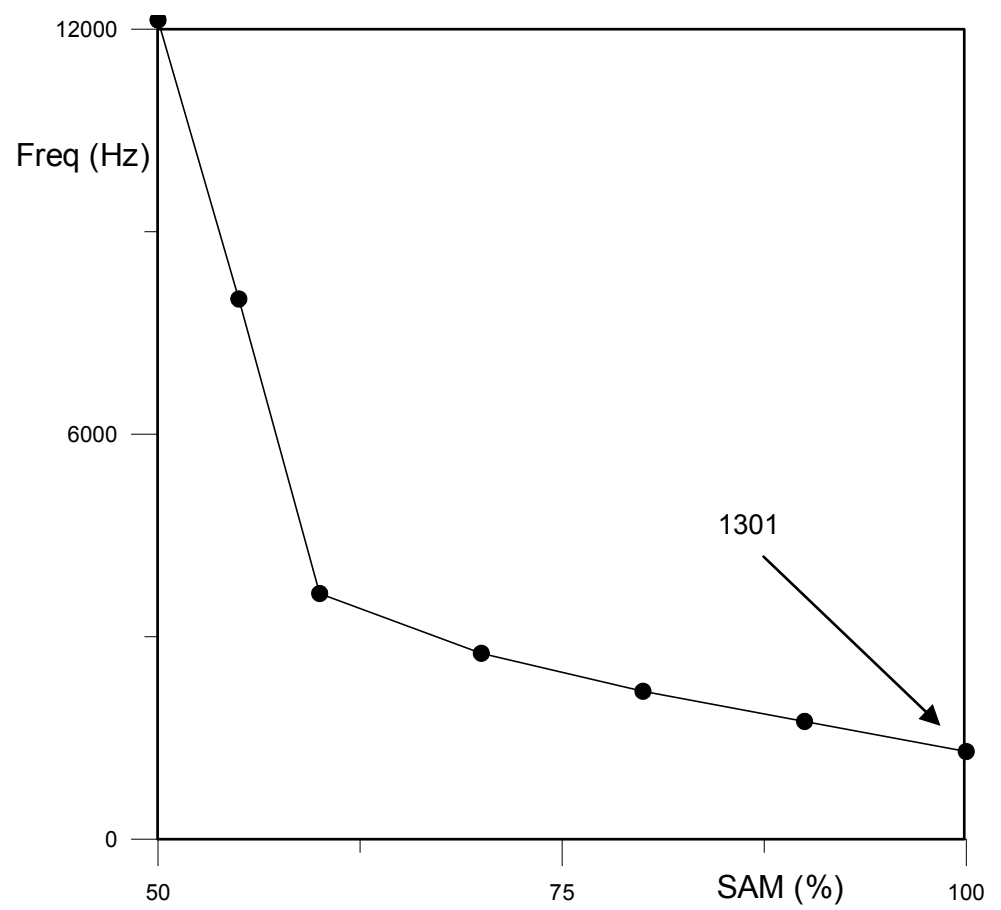

Figure 10: Characteristic spectral response of state of SAM coverage

\section{Conclusions}

The preliminary analysis carried out here shows that micro-dynamic characteristics of silicon micro-gear pairs change according to impact behaviour of their teeth. These characteristics depend on the impact velocity of their rough silicon teeth, which are influenced by adhesion of their asperities at lower gear set velocities, affecting rebound behaviour of contacts. At the usual high operational speeds this problem is almost negligible. With introduction of SAM, the problem of adhesion is almost entirely alleviated. With higher speeds, torque reversals become more significant and clearly there would exist an operational speed when the torque reversal would lead to oscillations of impacting pairs beyond the confine of their rigid separation (backlash), thus resulting in motion reversal of the gear set and loss of function.

A novel method is proposed to predict the degradation of the SAM protective coating on meshing gear teeth. This is a spectral-based approach which considers the changes in impact characteristics due to the depletion of SAM.

\section{References}

[1] Tanner D. M., Smith N. F., Irwin L. W., Eaton W. P., Helgesen K. S., Clement J. J., Miller W. M., Walraven J. A., Peterson K. A., Tangyunyong P., Dugger M. T., Miller S. L., (2000) "MEMS Reliability: Infrastructure, Test Structures, Experiments, and Failure Modes", SANDIA REPORT, SAND2000-0091

[2] Kim J. B., Choo H., Lin L. and Muller R. S., (2006) "Microfabricated Torsional Actuators Using Self-Aligned Plastic Deformation of Silicon", IEEE/ASME Journal of Microelectromechanical Systems, Vol. 15, No. 3, pp. 553-562, June

[3] Barlian A. A., Park S-J, Mukundan V. and Pruitta B. L., (2007) "Design and characterization of microfabricated piezoresistive floating element-based shear stress sensors", Sensors and Actuators A: Physical Volume 134, Issue 1, 28 February 2007, Pages 77-87

[4] Teodorescu M and Rahnejat H.: "Dry and Wet nano-Scale Impact Dynamics of Rough Surfaces with or without a Self-Assembled Mono-Layer": Proceedings of the Institution of Mechanical Engineers (IMechE), Journal of Nanoengineering and Nanosystems - Part N. in review 
[5] Teodorescu M. and Rahnejat H.: "Kinetic balance in nano-biotribological contact of gecko feet's spatulae": Proceedings of the Institution of Mechanical Engineers (IMechE), Journal of Nanoengineering and Nanosystems - Part N. in review

[6] Maboudian R., Ashurst W. R., Carraro C., (2000) "Self-assembled monolayers as anti-stiction coatings for MEMS: characteristics and recent developments“, Sensors and Actuators, Vol. 82, pp. 219-223

[7] Reedy Jr. E. D., Starr M. J., Jones R. E., Flater E. E., Carpick R. W., (2005) "Contact Modeling of SAMCoated Polysilicon Asperities" Proc. of the 28th Annual Meeting of the Adhesion Society Meeting, Mobile AL, Feb. 2005.

[8] Bhushan, B, Handbook of Micro/Nanotechnology, 2nd edition, CRC Press, Boca Raton, Florida, 1999.

[9] Dyck C. W., Smith J. H., Miller S. L., Russick E. M. and Adkins C. L. J., (1996) "Supercritical carbon dioxide solvent extraction from surface-micromachined micromechanical structures" SPIE Micromachining and Micro-fabrication, Oct. 1996.

[10] R. W. Gregory, S. L. Harris and R. G. Munro, (1963) "Dynamic behavior of spur gears" Proceedings of the Institution of Mechanical Engineers, 178, 1-28.

[11] H. N. Ozguven and D. R. Houser, (1988) "Mathematical models used in gear dynamics - a review" Journal of Sound and Vibration 1988, 121, 383-411.

[12] Theodossiades S. and Natsiavas S., (2001) "On geared rotordynamic systems with oil journal bearings". Journal of Sound and Vibration 2001, 243(4), 721-745.

[13] Greenwoon J. and Tripp J. H. (1970-17) "The contact of two Nominally Flat Rough Surfaces", Proc. Instn. Mech. Engrs., 185, 48/71 pp. 625-633

[14] Teodorescu M., Balakrishnan S. and Rahnejat H., (2006) "Physics of ultra-thin surface films on molecularly smooth surfaces" Proc. Instn. Mech. Engrs., J. Nano-Technology, 220 (1), 2006, pp. 7-19.

[15] Johnson K. L., Kendall K. and Roberts A. D., (1971) "Surface energy and the contact of elastic solids" Proc. Roy. Soc., Series A, 324 (1558) 1971, pp. 301-313.

[16] Johnson K. L. and Sridhar I., (2001) "Adhesion between a spherical indenter and an elastic solid with a compliant elastic coating" J. Phys. D: Appl. Phys. 34, 2001, pp. 683-689

[17] Fuller K. N. G. and Tabor D., (1975) "The Effect of Surface Roughness on the Adhesion of Elastic Solids" Proc. Roy. Soc., Series A, 345 (1642), 1975, pp. 327-342.

[18] B Derjaguin, K. L., Muller, V. M. and Toporov, Y. P. "Effect of contact deformation on the adhesion of particles" J. Colloid Interface Sci. 53, 314 (1971). pp.314-26

[19] Maugis, D., 1992, "Adhesion of Spheres: The JKR-DMT Transition Using a Dugdale Model," J. Colloid Interface Sci., 150, pp. 243-269.

[20] Johnson K L and Greenwood J A 1997 "An adhesion map for the contact of elastic spheres" J. Colloids Interface Sci. 192 326-33

[21] Tabor. D. (1977) "Surface forces and surface interactions" J. Colloids Interface Sci. 58, 1, 2-13.

[22] Johnson K. L. "Adhesion and friction between a smooth elastic spherical asperity and a plane surface", Proc. R. Soc. Lond. A (1997) 453, 163-179

[23] Hamrock B J., Fundamentals of Fluid Film Lubrication, McGraw-Hill International Editions, New York, London, New Mexico, 1994

[24] Starr M. J., Sumali H., Redmond J. M., Flater E. E. and Carpick R. W. "Analysis of Contact Forces Using AFM Data of Polycrystalline Silicon" Proc. of the SEM X International Congress and Exposition on Experimental and Applied Mechanics, Costa Mesa, CA, July 2004. 